\title{
Curso de física para biólogos, cambio de perspectiva en su enseñanza
}

\author{
P. Segarra, C. Alberto Villarreal, and D. N. Martínez \\ Departamento de Física, Facultad de Ciencias, UNAM, \\ Universidad 3000, Circuito Exterior s/n, 04510, Ciudad Universitaria, Ciudad de México. \\ *e-mail: psegarra@ciencias.unam.mx
}

Received 25 July 2021; accepted 19 August 2021

\begin{abstract}
En este trabajo se presentan las bases didácticas que guiaron la organización y elaboración de materiales en un curso de física para biólogos, de acuerdo con el programa vigente de la Facultad de Ciencias, UNAM, para dicha asignatura. El curso se diseñó con base a los resultados en la investigación sobre la enseñanza de la física ${ }^{i}$, lo que llevó a implementar en la dinámica cotidiana la modelización, contextualización, desarrollo de lo concreto a lo formal, trabajo activo de los alumnos tanto de forma individual como en grupos pequeños. Se presentan los resultados obtenidos, al aplicarlo a lo largo de 6 semestres (2016-I a 2019-I), con grupos tanto de primer ingreso como de repetidores. La población total consta de 128 estudiantes, constituida el $70 \%$ por mujeres.
\end{abstract}

Descriptores: Diseño de curso; física universitaria introductoria; enseñanza de la física; actitud; motivación; género.

In this work didactic principles that guided the organization and processing of materials in a physics course for biologists, according to the current program of the College of Science, UNAM, for that subject are presented. The course was designed based on the results in physics education research, which led to the implementation in the daily dynamics of modeling, contextualization, development of the concrete to the formal and active work of students both individually as in small groups. The results apply to the over 6 semesters (2016-I to 2019-I) with groups both freshmen and repeating students. The total population consists of 128 students from which $70 \%$ are women.

Keywords: Curse design; introductory university physics; attitude; motivation; gender.

PACS: 01.40.Di; 01.40.Fk

DOI: https://doi.org/10.31349/RevMexFisE.18.020211

\section{Antecedentes}

Con anterioridad a este nuevo diseño, una de las autoras, había impartido la materia Física para biólogos con el programa actual y con el programa anterior durante 14 semestres. Por lo tanto, se podía suponer un conocimiento de los intereses, fortalezas y debilidades de la población que llega a este curso. La mayor dificultad percibida a lo largo de estos 14 semestres previos era el rechazo que muestran los estudiantes de la carrera de biología hacia la física, junto con la creencia de que son incapaces de aprenderla. Además, afirman que no necesitan la física para sus estudios de biología ni para trabajar posteriormente como biólogos, lo cual se ve reflejado en el programa actual (plan 1997), al que se disminuyó la carga horaria a la mitad respecto del anterior. Esta actitud es más generalizada a nivel mundial de lo que pudiera esperarse, como puede verse en la literatura [1-3].

El primer punto, al tomar nuevamente el curso de física para biólogos en 2015, fue reflexionar seriamente qué física requiere saber esta población y qué dificultades tienen para el aprendizaje. Se supuso como punto de partida que el profesional de la biología debe tener un conocimiento conceptual profundo, que permita la interpretación de fenómenos en contextos distintos, por lo que evidentemente no basta la memorización y la resolución de problemas algorítmicos. Este punto lo desarrolla ampliamente Hodson [4], aunque no se refiere a universitarios, cuando señala que el currículo científico no cubre las necesidades y aspiraciones de los jóvenes que requieren tener un conocimiento científico que pueda ser utilizado para llegar a conclusiones basados en la evidencia; así como adquirir la capacidad y el compromiso de tomar medidas apropiadas, responsables y efectivas en asuntos de interés social, económico, ambiental y ético-moral. Los principales problemas por resolver con el planteamiento actual del curso son: abatir el desinterés y mostrar a los estudiantes que tienen la capacidad para aprender y entender la física. Se sabe que el autoconcepto actúa como un predictor en el rendimiento académico y por ello, es de suma importancia el refuerzo positivo $[5,6]$.

La idea de esta investigación fue documentar lo que sucede en el aula al aplicar lineamientos derivados de la investigación en el área. Promovemos conscientemente: la relación de la física con la biología, el trabajo en situaciones problemáticas que permiten integración del trabajo teórico y práctico, la construcción de modelos explicativos que puedan irse acercando al modelo científico correspondiente, un ambiente positivo de aula, el trabajo en equipos pequeños y en grupo, el refuerzo positivo hacia preguntas $\mathrm{y}$ aportaciones (especialmente cuando muestran un proceso metacognitivo), expectativa de éxito hacia cada estudiante, apoyos puntuales en matemáticas cuando se detectan dificultades en algún tema, actividades lúdicas y el gusto por la asignatura por parte de los académicos. El itinerario que se sigue es de lo concreto y evidente a lo abstracto; se guía a los grupos de trabajo para que analicen las observaciones y resultados, para que valoren la importancia de controlar variables, para que ellos empiecen a construir modelos explicativos que les permitan predecir. Deben convencerse de que este acercamiento hacia la física y esta forma de aprenderla es mucho 
más útil que únicamente manipular fórmulas y obtener resultados sin sentido.

La propuesta de Cordero [7], basada también en la investigación educativa, se asemeja a la que se está desarrollando, en muchos puntos, pero debido a los más de 20 años que median en la investigación necesariamente se difiere en el aspecto de conflicto cognitivo y choque conceptual que actúan en contra del autoconcepto. En las investigaciones del área de didáctica de la física hemos transitado de las ideas previas y cambio conceptual a la construcción de modelos por parte de los estudiantes [8-10].

Una pregunta importante cuando nos enfrentamos a un curso para estudiantes de biología es ¿este curso es distinto que el que les daría a los físicos o a los ingenieros? En estudios realizados en Estados Unidos referidos a ciencias biológicas y de la salud se afirma que no se debe dar física de la misma manera a estudiantes de física e ingeniería que a estudiantes de biología y ciencias de la salud, ya que los intereses profesionales son distintos [2,11-14], a este mismo resultado se llegó en México en un estudio de 5 años con estudiantes de área II (Químico-Biológicas) de la Escuela Nacional Preparatoria [15].

Este primer planteamiento muestra un giro del estar centrados en la asignatura a preguntarnos por lo que puede ser de utilidad para los alumnos. Con base en los intereses de la población se recalca que los cursos para biólogos, físicos, ingenieros o químicos evidentemente deberían ser distintos y que la contextualización de los temas en las áreas de interés o estudio de los alumnos es de suma importancia. Se sabe que las clases tradicionales no dan los mejores resultados de aprendizaje. En esta categoría se incluyen las clases magistrales que tienen al profesor como principal actor y a la lógica de la disciplina como forma de organización. La investigación en enseñanza de las ciencias propone cambiar la organización de la clase a una forma participativa, de preferencia en grupos pequeños, donde el estudiante tenga un papel activo [16-18]. En muchos casos el aprendizaje más profundo se da en el momento en el que tratan de expresar a otro compañero en voz alta lo que entendieron respecto del tema o fenómeno.

\section{Población con actitud negativa hacia el aprendizaje de la física}

La literatura sobre enseñanza de la física apunta que el desinterés de los estudiantes va aumentando con la edad [1,3,9-22] y también asienta que el desinterés es mayor en las niñas que los niños; es más, se suele decir física para los niños y biología para las niñas $[1,23,24]$. Ante estos datos surge la pregunta ¿por qué este desinterés y por qué mayor en las niñas que en los niños? Parece ser que parte del problema está en los ejemplos utilizados, muchos estudios señalan que éstos, tienen un sesgo de género, lo que resulta que los muchachos tengan mejores resultados en general que las muchachas.

Se sabe que las mujeres tienen más interés en cuestiones relacionadas con la salud y los seres vivos y que para los hombres ejemplos de coches, choques y aviones pueden ser interesantes. Entonces, los ejemplos que estamos utilizando en física aparentemente hacen que las niñas de secundaria y bachillerato no se interesen en la asignatura y opten por estudiar algo más relacionado con ciencias biológicas o de la salud. En la carrera de biología la mayor parte de la población es femenina. En los 6 grupos documentados para este estudio la relación es 7 a 3 por cada 10 estudiantes. Este dato sería suficiente para suponer que la población de estudiantes de biología no tiene interés en el aprendizaje de la física. En el estudio de Arandia [1] se afirma que "estudiantes de biología muestran un nivel de motivación bajo e inferior al resto"; donde el resto se refiere a estudiantes de bachillerato, ingeniería y física.

El desinterés hacia la física es aún mayor en los grupos de repetidores, que en los alumnos de primer ingreso. Además del fracaso sufrido, muchos estudiantes avanzan en la carrera sin aprobar física, lo que refuerza la idea de que no la necesitan. En la muestra utilizada en este estudio, 5 estudiantes en un grupo de 23 (en el semestre del 2018) estaban cursando el último semestre, aunque la mayoría, 16, eran de segundo semestre.

El dato queda confirmado en el documento para la revisión del plan de estudios para la carrera de Biología de la Facultad de Ciencias de la UNAM, donde se muestra una opinión poco favorable hacia la aportación de la asignatura de física en la formación de los futuros biólogos, por lo cual se busca retirarla del plan de estudios.

\section{Descripción de la propuesta}

El curso se diseñó con la finalidad de hacer la física más cercana a los estudiantes de biología, mediante la contextualización. El primer objetivo del curso ha sido quitar el odio hacia la física y el segundo, mostrar cómo los modelos físicos estudiados en el programa se relacionan con fenómenos biológicos y pueden servir para entender mejor esta ciencia [25]. Se recalca que el interés de esta población está en saber biología y los estudiantes entran a la carrera pensando que la física no tiene relación alguna con ésta. En el curso que se está describiendo se tiene el propósito de que los alumnos sean capaces de utilizar sus modelos como "criterio de verdad" [26] para evaluar si algunos cálculos realizados en clase tienen sentido.

El maestro puede hacer sus mejores esfuerzos, pero si no logra que los estudiantes se interesen en la disciplina, se motiven para su estudio y tengan el compromiso de trabajar en la misma, los resultados pueden ser prácticamente nulos. Esto significa que como maestros nos debemos centrar en el aprendizaje de nuestros alumnos, no sólo en la enseñanza. El lograr cautivarlos en un primer momento no significa que los podemos tener interesados a lo largo del semestre por lo que es necesario estar recurriendo a nuevos elementos que puedan captar la atención en diferentes momentos y así poder involucrarlos en lo que se quiere que hagan para favorecer aporta- 
ciones desde su experiencia como estudiantes de biología y finalmente lograr los aprendizajes esperados [27-30].

Todas las sesiones en el curso de física para biólogos se detonan con un tema que puede ser interesante para los estudiantes de biología, por lo que se ha requerido que los profesores estudiemos a fondo varios fenómenos de tipo biológico. En el desarrollo de los temas juega un papel muy importante, el trabajo práctico, la modelización de los fenómenos físicos y el poder explicativo y predictivos de los modelos construidos, así como su límite de validez. En el curso se manejan TICs, sólo como un medio para enriquecer la comunicación entre los miembros del grupo, compartir materiales y ajustarnos a la dinámica que tienen los estudiantes actuales que ya nacieron con las tecnologías. Se utiliza una red social con un grupo cerrado donde se comparten simulaciones, se les ponen las guías de las prácticas, tareas, y los estudiantes envían sus trabajos por medio de un mensaje. Inicialmente se utilizó una plataforma educativa pero no dio resultado, en contraste con la red social que ha mostrado ser muy exitosa.

En algunas sesiones del semestre se utiliza el aula invertida, donde antes de llegar a la discusión en clase, los estudiantes deben haber visto un vídeo, trabajado con un simulador o realizado una lectura [31-33]. La Tabla I muestra la organización en tres unidades, así como las ideas claves y las aplicaciones con las que se relacionan. El temario cubierto durante el semestre está apegado al programa oficial, pero en cada tema se ha hecho una relación explícita con la biología, esto ha obligado a dejar fuera dos unidades señaladas en el programa (fuerzas y el universo) dado que son las que menos carga horaria tienen y su objetivo es el inicio y cierre del curso, que es cubierto de otra manera. El sacrificar estas dos unidades del programa ha permitido dedicar 10 sesiones a cada una de las otras tres, haciendo énfasis en la contextualización, que es uno de los ejes principales de este trabajo.

El curso de física para biólogos, en la Facultad de Ciencias, UNAM, tiene una duración de 16 semanas, con una car-

TABLA I. Organización de las unidades con ideas clave y aplicaciones.
Unidad 1: Óptica, 10 sesiones Ideas clave: lentes y espejos; refracción y reflexión. Aplicaciones a ojo, microscopio y telescopio.
Unidad 2: Ondas y modelos atómicos, 10 sesiones Ideas clave: ondas mecánicas y electromagnéticas, características de las ondas, color, espectros absorción y emisión.
Aplicaciones a percepción remota, espectroscopios, terremotos, música, ecorreceptores.

Unidad 3: $\quad$ Electromagnetismo, 10 sesiones Ideas clave: campo eléctrico, diferencia de potencial, carga, corriente, resistencia, circuitos, magnetismo. Aplicaciones: impulso nervioso, electrorreceptores en tiburones y anguila eléctrica, migración. ga de 6 horas a la semana, distribuidas en dos clases que se imparten en un laboratorio, a un máximo de 25 estudiantes, lo que facilita la movilidad de ellos en el aula y el trabajo grupal. Para la realización de la mayor parte de las actividades, se trabaja en equipos pequeños, usualmente de 3 estudiantes, aunque no se ha descuidado el trabajo individual. La experiencia muestra mejores resultados en los equipos de tres que cuando es necesario utilizar un equipo de dos o de cuatro estudiantes. Cabe señalar que en cada semestre se han introducido modificaciones surgidas de las inquietudes de los docentes o de propuestas de los estudiantes. En este trabajo se describe el marco común en los 6 semestres.

En las sesiones no hay una distinción rígida entre el trabajo práctico, resolución de problemas, realización de experimentos y el momento de la clase teórica, ya que las explicaciones se van dando durante el desarrollo de las actividades [34]. Este enfoque ha permitido que los estudiantes piensen los problemas físicamente, de manera cualitativa, antes de lanzarse a realizar sustituciones. Un ejercicio continuo en clase es la pregunta de si los resultados obtenidos, por medio de cálculos, concuerda con el modelo conceptual generado por cada estudiante.

Llama la atención la similitud de los ejemplos con los propuestos por Kortmeyer [35] para el segundo semestre de física, aunque esta propuesta está referida directamente a estudiantes de medicina. En contraposición, los cursos para poblaciones similares en universidades de Estados Unidos son anuales o dos cursos semestrales, con una carga horaria similar, por lo que duplican la que nosotros tenemos.

\section{Muestra}

La población descrita en este trabajo consta de 128 estudiantes de la carrera de biología repartidos en 4 grupos de primer semestre y 2 de repetidores, de los cuales 92 (72\%) son mujeres y $36(28 \%)$ hombres. Los alumnos de primer ingreso son asignados a los distintos grupos por sorteo, por lo que se pueden considerar representativos de la población. Los repetidores eligen el grupo donde quieren inscribirse, esta elección está regida usualmente por el horario o por el profesor que imparte la materia. El primer día de clase se pone un cuestionario inicial donde entre otras cosas se les pregunta si piensan que la física tiene utilidad a lo largo de su carrera, la respuesta de la mitad de los estudiantes es NO. Un resultado similar se encuentra en el estudio de Kortemeyer [35] referido a estudiantes de medicina.

\section{5. ¿Por qué podemos decir que estamos te- niendo éxito con este curso?}

\subsection{Cuestionario de opinión}

En el cuestionario de entrada (A1), $60 \%$ afirma no estar interesado en la física porque no le entienden a las matemáticas, y $32 \%$ dicen que los profesores que han tenido son confusos 


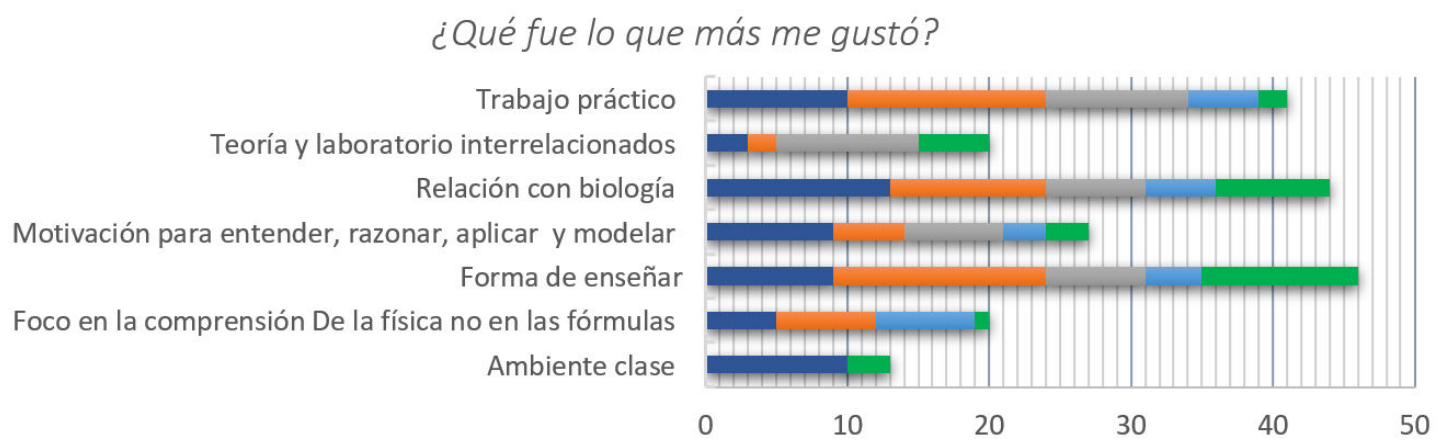

GRÁFICA 1. Opinión de los estudiantes sobre la propuesta.

y tediosos. Más de la mitad piensa que la física no tiene relación alguna con la biología, agravado en los repetidores que están terminando la carrera.

Al finalizar el curso se realiza un cuestionario con preguntas abiertas (A2), respecto a lo que más les gustó (Gráfica 1). Entre las opiniones resalta: la forma de enseñar, la relación física/biología y el trabajo práctico. Cabe mencionar que no fue posible recabar los datos del semestre 2018-1 debido al sismo del 2017.

Ante la pregunta sobre qué modificarían en el curso, el $66 \%$ responde "nada", y a la de qué añadirías llama la atención que, $16 \%$ del total, solicitan otro curso de física donde se aborde fluidos, termodinámica y mecánica, principalmente en los dos grupos de repetidores. Estos son los mismos estudiantes que en el cuestionario inicial afirmaron que la física no estaba relacionada con la biología. Con los repetidores, los comentarios espontáneos en algunas sesiones eran: si esto lo hubiera sabido antes me hubiera ido mejor en molecular; con esto hubiera comprendido mejor lo del contraste de fases; fisiología animal hubiera sido distinta si yo hubiera sabido qué es la diferencia de potencial, así como armar e interpretar el funcionamiento de circuitos; ya entiendo porque tengo que utilizar diferentes espectroscopios en los análisis. Esta gran diferencia entre los grupos de repetidores y los de primer ingreso requiere un análisis más amplio, que queda fuera de la intención de este artículo de describir de forma general la dinámica introducida.

Finalmente, respecto al cuestionario de opinión cuando se les pide añadir cualquier otro comentario surgen algunos como: nos hicieron ver la física con otros ojos, nos hicieron amar la física, ya me gusta la física y gracias por hacer que entendiera la física.

Estos resultados indican que al menos se logró cambiar la aversión hacia la física por un gusto puntual. Hay muchos comentarios en la dirección de que es una clase a la que se asiste con gusto. El resultado tangible es una baja deserción y una asistencia alta en todas las sesiones. Otro indicador de éxito es que en los semestres en el que los estudiantes pueden elegir profesor para la asignatura, el cupo para el curso se llenó el primer día de inscripciones. La pregunta obligada es ¿aprendieron algo, además de que les gustó la clase?

\subsection{Desempeño del grupo experimental}

En la primera aplicación de esta propuesta se tuvo una excelente respuesta de los estudiantes. Hubo alguna deserción o estudiante que nunca se presentó, pero todos los que terminaron el semestre obtuvieron un resultado aprobatorio. A falta de una estadística de lo que ocurría históricamente con esta asignatura se hizo una primera comparación de nuestro grupo experimental con los datos de 22 grupos de la misma materia (Gráfica 2).

El contraste tan alto de nuestros resultados con el promedio de los otros grupos nos llevó a cuestionarnos si la diferencia en la calificación se debía a la facilidad de los exámenes, o al enfoque y dinámica del curso.

Esta fue la razón por lo que, para verificar la comprensión conceptual de nuestros estudiantes, se recurrió paulatinamente a incorporar dentro de la dinámica de la case, algunas preguntas tomadas de artículos de investigación, donde se señalan problemas específicos de aprendizaje y así poder tener un patrón de comparación.

Todos estos artículos tienen un anexo con el instrumento utilizado para detectar los problemas de aprendizaje. Varias de estas preguntas fueron empleadas como parte de la discusión en clase, trabajo en grupos pequeños, guía de prácticas y en algún caso como pregunta de examen.

\section{Rendimiento académico semestre 2016-1}

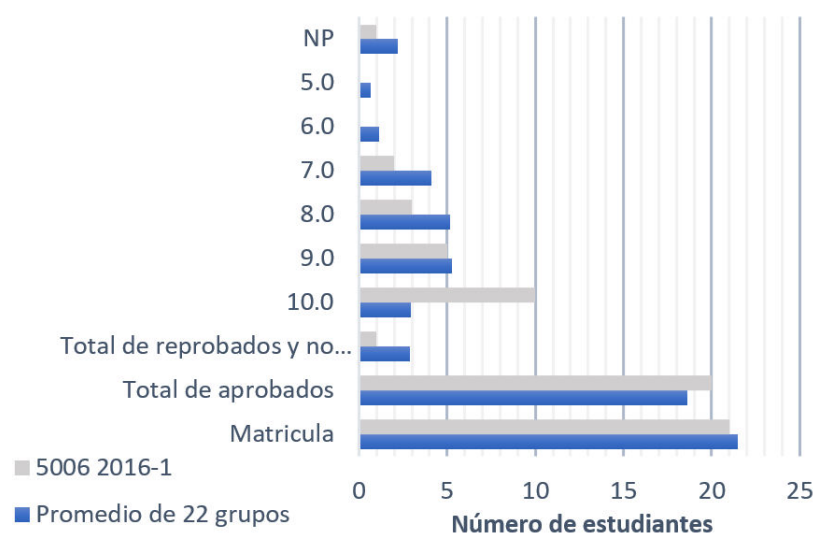

GRÁFICA 2. Comparación del grupo experimental con el promedio de 22 grupos de la misma asignatura. 


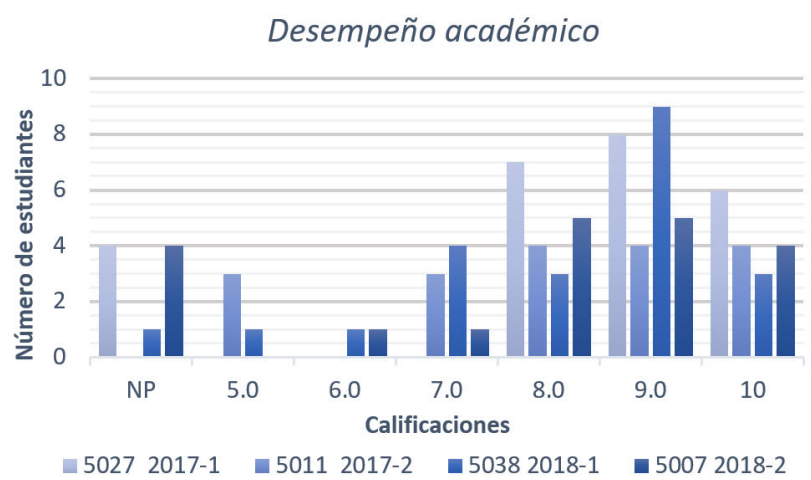

GRÁFICA 3. Calificaciones obtenidas por los estudiantes de primer ingreso y repetidores en dos años consecutivos.

Tomando como referencia estas comparaciones, se puede concluir que los aprendizajes logrados muestran comprensión del tema. En otros trabajos se profundizará sobre los resultados específicos en cada uno de los temas.

Algunos de los artículos utilizados para poder comparar nuestros resultados son: Un estudio sobre «qué, cuándo y cuánto $\gg$ aprenden los alumnos acerca de la visión [36], Representaciones de alumnos universitarios sobre propagación de ondas mecánicas [37], Obstáculos para aprender conceptos elementales de electrostática y propuestas educativas [38], Dificultades de aprendizaje de los conceptos de carga y campo eléctrico en estudiantes de bachillerato y universidad [39], La enseñanza del concepto de campo eléctrico basada en un modelo de aprendizaje como investigación orientada [40], Persistencia de las ideas previas sobre potencial eléctrico, intensidad de corriente y ley de $\mathrm{Ohm}$ en los estudiantes de segundo curso de Ingeniería [41].

Conviene señalar que se obtienen mejores resultados con los alumnos de primer semestre que con los repetidores. Lo cual se ve reflejado en la Gráfica 3 donde los grupos de recursadores en los que se han aplicado esta propuesta, se aprecia una diferencia en las calificaciones con respecto a las obtenidas por los estudiantes de nuevo ingreso. Esto nos habla de factores intricados de rechazo al aprendizaje, ya que los estudiantes se encuentran más interesados en aprender mediante memorización lo necesario para acreditar el curso que en la comprensión de los conceptos de la materia, tema que forma parte de otra investigación.

\section{Conclusiones}

¿Cuál es el factor más importante para los resultados obtenidos, en medio de esta cantidad de estrategias y actividades? De acuerdo con Mellado [42], un factor muy importante es la actitud y dedicación de los docentes junto con el ambiente empático que se logra en la clase. Desde el primer día en el curso se propicia la participación de los estudiantes al quitarles el anonimato. Para lograr este objetivo, se requiere de una atención cercana a todos los equipos de trabajo, por ello es necesario que se encuentren presentes en todas las sesiones los profesores de teoría y laboratorio, así como la ayudante; esto supone una atención por encima del promedio de otras asignaturas.

La intención del curso ha sido que los egresados de la materia puedan percibir la utilidad de la física (conceptos, herramientas y métodos) en la biología y que por lo tanto cambie su disposición hacia el aprendizaje de esta. A través de los aprendizajes logrados y cuestionarios de opinión validamos el logro de este propósito.

Los alumnos de primer ingreso se extrañan de la posibilidad de aplicar la física a su futura carrera, lo cual les despierta interés y los alienta a profundizar en los temas, logrando aprendizajes significativos. Los recursadores, al estar varios terminando la carrera, aportan ejemplos biológicos donde les hubiera ayudado tener mejores bases físicas para comprenderlos.

El énfasis de centrarnos en describir la población a la que se dirige el curso, en su mayoría es femenina, es porque en la literatura se ha reportado que presentan mayor dificultad en los aprendizajes de física debido al tipo de ejemplos con lo que se trabajan. La contextualización en la biología permitió capturar el interés de la población femenina para alcanzar los aprendizajes esperados. Cabe resaltar que estos ejemplos fueron de interés también para la población masculina ya que se trabajaron temas relacionados a su carrera.

En los cursos siguientes al 2019 -1 se ha continuado con este enfoque, pero ahora trabajando con dos grupos para llegar a mayor población. Esta propuesta puede aplicarse para otras disciplinas poniendo atención en la contextualización que pueda interesar a estos estudiantes. La experiencia obtenida con los biólogos está sirviendo como basa para realizar la propuesta del programa del curso "Óptica y Acústica" de quinto semestre para la Licenciatura en Enseñanza y Aprendizaje de la Física en Educación Secundaria. Este programa forma parte de la reforma 2018 para la formación de profesores de secundaria, de lo que anteriormente se llamaban escuelas normales.

\section{Anexo 1: Cuestionario Inicial}

1. Nombre completo

2. ¿Cómo te gusta que te digan? (apodos no)

3. Realiza una breve descripción sobre ti

4. ¿Cuáles son tus pasatiempos?

5. Escuela de procedencia

6. ¿Fue biología tu primera opción?

7. En caso de que tu respuesta anterior sea no, escribe cual fue tu primera opción.

8. ¿Por qué elegiste biología?

9. ¿En qué áreas de la biología estas interesado?

10. ¿Consideras que tienes dificultades con física y matemáticas? 
11. ¿Qué piensas sobre la física?

12. Escribe que temas de física viste en el bachillerato

13. ¿Qué relaciones explícitas viste en los cursos anteriores entre la física y la biología?

\section{Anexo 2: Cuestionario de opinión final}

1. ¿Qué fue lo que más me gusto del curso de física?
2. ¿Qué quitaría?

3. ¿Qué añadiría?

4. ¿Qué consideras que fue lo más difícil o qué se te dificultó más?

5. Cualquier otro comentario
1. E. Arandia, K. Zuza y J. Guisasola, Actitudes y motivaciones de los estudiantes de ciencias en Bachillerato y Universidad hacia el aprendizaje de la Física, Rev. Eureka Enseñ. Divulg. Cienc. 13 (2016) 558, https: / hdl . handle.net/ $10498 / 18497$

2. C. H. Crouch y K. Heller, Introductory physics in biological context: An approach to improve introductory physics for life science students, Am. J. Phys. 82 (2014) 378, https: //doi.org/10.1119/1.4870079

3. J. Osborne, S. Simon y S. Collins, Attitudes towards science: A review of the literature and its implications, Int. J. Sci. Educ. 25 (2003) 1049, https: / / doi.org/10.1080/ 0950069032000032199

4. D. Hodson, Time for action: Science education for an alternative future, Int. J. Sci. Educ. Int. J. Sci. Educ. (2003) 645, https://doi.org/10.1080/09500690305021.

5. S. Costa y C. Tabernero, Rendimiento académico y autoconcepto en estudiantes de educación secundaria obligatoria según el género, Rev. Iberoam. Psicol. Salud J. Sci. Educ. (2012) 175.

6. D. Cheung, The key factors affecting students' individual interest in school science lessons, Int. J. Sci. Educ. Rev. Iberoam. Psicol. Salud (2018) 1, https://doi.org/10.1080/ 09500693.2017 .1362711

7. S. Cordero, D. Petrucci y A. Dumrauf, Enseñanza Universitaria de Física: ¿En un taller?, Rev. Enseñ. Fis. 9 (1996) 14.

8. R. Duit y D. F. Treagust, Conceptual change: A powerful framework for improving science teaching and learning, Int. J. Sci. Educ. 25 (2003) 671, https://doi.org/10.1080/ 09500690305016

9. I. T. Koponen, Models and Modelling in Physics Education: A Critical Reanalysis of Philosophical Underpinnings and Suggestions for Revisions, Sci. Educ. 16 (2007) 751, https: //doi.org/10.1007/s11191-006-9000-7

10. P. S. Oh y S. J. Oh, What Teachers of Science Need to Know about Models: An Overview, Int. J. Sci. Educ. 33 (2011) 1109, https://doi.org/10.1080/09500693. 2010.502191

11. C. H. Crouch y K. Heller, Teaching physics to life science students - Examining the role of biological context, AIP. Conf. Proc. 1413 (2012) 159, https: / /doi.org/10.1063/1. 3680019

12. A.-M. Hoskinson, B. A. Couch, B. M. Zwicki, K. A. Hinko y M. D. Caballero, Bridging physics and biology teaching through modeling, Am. J. Phys. 82 (2014) 434, https:// doi.org/10.1119/1.4870502

13. D. C. Meredith y E. F. Redish, Reinventing physics for lifesciences majors, Phys. Today 66 (2013) 38, https: //doi. org/10.1063/PT.3.2046.

14. V. Sawtelle y C. Turpen, Leveraging a relationship with biology to expand a relationship with physics, Phys. Rev. Phys. Educ. Res. 12 (2016) 010136, https://doi.org/10.1103/ PhysRevPhysEducRes.12.010136.

15. A. Flores, Uso del enfoque CTS para la enseñanza de la física en el área de las ciencias biológicas y de la salud, Tesis de maestría, UNAM, 2013, http://132.248.9. 195/ptd2013/noviembre/0705601/Index.html.

16. Y. Benítez y C. Mora, Enseñanza tradicional vs aprendizaje activo para alumnos de ingeniería, Rev. Cub. Fís. 27 (2010) 175, https://www.repositoriodigital.ipn.mx/ handle/123456789/10702.

17. A. Marzábal, A. Rocha y B. Toledo, Caracterización del desarrollo profesional de profesores de ciencias-parte 2: Proceso de apropiación de un modelo didáctico basado en el ciclo constructivista del aprendizaje, Educ. Quím. 26 (2015) 212, https://doi.org/10.1016/j.eq.2015.05.006

18. C. Henderson, R. Khan y M. Dancy, Will my student evaluations decrease if I adopt an active learning instructional strategy?, Am. J. Phys. 86 (2018) 934, https://doi.org/10. $1119 / 1.5065907$

19. A. Vázquez y M. A. Manassero, El declive de las actitudes hacia la ciencia de los estudiantes: un indicador inquietante para la educación científica, Rev. Eureka Enseñ. Divulg. Cienc. 8 (2008) 274, https://doi.org/10.25267/ Rev_Eureka_ensen_divulg_cienc.2008.v5.i3.03

20. N. Reid y E. A. Skryabina, Attitudes towards Physics, Res. Sci. Technol. Educ. 20 (2002) 67, https : / / doi.org/10. $1080 / 02635140220130939$

21. J. Solbes, R. Montserrat y C. Furió, El desinterés del alumnado hacia el aprendizaje de la ciencia: implicaciones en su enseñanza, Didact. Cienc. Exp. Soc. 21 (2007) 91, https://ojs. uv.es/index.php/dces/article/view/2428

22. C. Williams, M. Stanisstreet, K. Spall, E. Boyes y D. Dickson, Why aren't secondary students interested in physics?, Phys. Educ. 38 (2003) 324, https://doi.org/10.1088/ $0031-9120 / 38 / 4 / 306$ 
23. A. Baram-Tsabari y A. Yarden, Girls' biology, boys' physics: evidence from free-choice science learnin setting, Res. Sci. Technol. Educ. 26 (2008) 75, https://doi.org/10. $1080 / 02635140701847538$

24. J. C. Blickenstaff, Women and science careers: leaky pipeline or gender filter?, Gend. Educ. 17 (2005) 369, https : / / doi. org/10.1080/09540250500145072

25. P. Segarra y C. A. Villareal, Circuito DC como modelo análogo del axón, Enseñ. Cienc. Extra (2017) 4351, https://raco.cat/index.php/Ensenanza/ article/view/337624

26. R. Gutiérrez, Mental Models and the Fine Structure of Conceptual Change, en Physics Teacher Education Beyond 2000, editado por R. Pinto y S. Surinach (Elsevier, Paris, 2001), pp. 35-44.

27. P. Potvin y A. Hasni, Interest, motivation and attitude towards science and technology at K-12 levels: a systematic review of 12 years of educational research, Stud. Sci. Educ. 50 (2014) 85, https://doi.org/10.1080/03057267. 2014.881626

28. S. Hidi y J. M. Harackiewicz, Motivating the Academically Unmotivated: A Critical Issue for the 21st Century, Rev. Educ. Res. 70 (2000) 151, https: //doi.org/10.3102/ 00346543070002151

29. A. Loukomies et al., Promoting Student's Interest and Motivation Towards Science Learning: the Role of Personal Needs and Motivation Orientations, Res. Sci. Educ. 43 (2013) 2517, https://doi.org/10.1007/s11165-013-9370-1

30. J. Bennett, S. Hogarth y F. Lubben, A systematic review of the effects of context-based and Science-Technology-Society (STS) approaches in the teaching of secondary science, en Research Evidence in Education Library (EPPI-Centre Institute of Education, Londres, 2003), p. 128.

31. J. L. Bishop y M. A. Verleger, The Flipped Classroom: A Survey of the Research, en Proceedings of the 120th ASEE Annual Conference and Exposition, Atlanta, 2013, editado por American Society for Engineering Education (ASEE, Atlanta, 2013), Vol. 30, https: //doi .org/10.18260/1-2--22585.

32. L. Abeysekera y P. Dawson, Motivation and cognitive load in the flipped classroom: definition, rationale and a call for research, High Educ. Res. Dev. 34 (2015) 1, https://doi. org/10.1080/07294360.2014.934336

33. B. Tucker, The Flipped Classroom, Educ. Next 12 (2012) 82, https://www.educationnext.org/ the-flipped-classroom/
34. A. Garritz Ruiz y G. Irazoque Palazuelos, El trabajo práctico integrado con la resolución de problemas y el aprendizaje conceptual en la química de polímeros, Didact. Cienc. Exp. 39 (2004) 40, https://andoni.garritz.com/documentos/ alambique39_trabajos_practicos.pdf.

35. G. Kortemeyer, The Challenge of Teaching Introductory Physics to Premedical Students, Phys. Teach. 45 (2007) 552, https://doi.org/10.1119/1.2809149

36. B. Bravo, M. Pesa y J. Pozo, La Enseñanza y el aprendizaje de las ciencias. Un estudio sobre «qué, cuándo y cuánto $\gg$ aprenden los alumnos acerca de la visión, Enseñ. Cienc. 30 (2012) 109, https: / / raco. cat/index.php/ Ensenanza/article/view/285686

37. S. Bravo, M. Pesa y M. C. Caballero Sahelices, Representaciones de alumnos universitarios sobre propagación de ondas mecánicas, Enseñ. Cienc. 27 (2009) 405, https://raco.cat/index.php/Ensenanza/ article/view/142073

38. A. Criado y P. Cañal, Obstáculos para aprender conceptos experimentales de electrostática y propuestas educativas, Inv. Esc. 47 (2002) 53, https://revistascientificas. us.es/index.php/IE/article/view/7621

39. C. Furió y J. Guisasola, Dificultades de aprendizaje de los conceptos de carga y de campo eléctrico en estudiantes de bachillerato y universidad, Enseñ Cienc. 16 (1998) 131, https://raco.cat/index.php/Ensenanza/ article/view/83239.

40. C. Furió y J. Guisasola, La enseñanza del concepto de campo eléctrico basada en un modelo de aprendizaje como investigación orientada, Enseñ. Cienc. 19 (2001) 319, https://raco.cat/index.php/Ensenanza/ article/view/21750

41. M. C. Periago Oliver y X. Bohigas Janoher, Persistencia de las ideas previas sobre potencial eléctrico, intensidad de corriente y ley de Ohm en los estudiantes de sgundo curso de Ingeniería, Rev. Electron. Investig. Educ. 7 (2005) 1, https: //www.redalyc.org/articulo.oa?id=15507207

42. V. Mellado, A. Garritz y M. Brígido, La dimensión afectiva olvidada del conocimiento didáctico del contenido de los profesores de ciencias Extra (2009) 347, https://raco.cat/ index.php/Ensenanza/article/view/293476 